\title{
A teoria cantoriana dos números transfinitos: sua relação com o pensamento analógico-geométrico
}

\author{
Cantorian Theory of Transfinite Numbers: \\ Its Relation with Analogical-Geometric Thought
}

*Walter Gomide

\begin{abstract}
Resumo: Neste pequeno artigo, analiso como a intuição geométrica estava presente no desenvolvimento seminal da teoria cantoriana dos conjuntos. Deste fato, decorre que a noção de conjunto ou de número transfinito não era tratada por Cantor como algo que merecesse uma fundamentação lógica. Os paradoxos que surgiram na teoria de Cantor são fruto de tal descompromisso inicial, e as tentativas ulteriores de resolvê-los fizeram com que aspectos intuitivos e esperados sobre os conjuntos ou infinito se perdessem. Em especial, observa-se aqui as consequências "não geométricas" do axioma da construtibilidade de Gödel.
\end{abstract}

Palavras-chave: Teoria dos conjuntos. Intuição geométrica. G. Cantor. Axioma de construtibilidade. K. Gödel.

Abstract: In this short article, I analyze how geometric intuition was present in the seminal development of Cantor's set theory. From this fact, it follows that the notion of set or transfinite number was not treated by Cantor as something worthy of a logical foundation. The paradoxes that have arisen in Cantor's theory are the result of such initial disengagement, and subsequent attempts to solve them have resulted in intuitive and expected aspects on sets or the infinite being lost. In particular, we see here the "non geometric" consequences of Gödel's axiom of constructible sets.

Keywords: Set theory. Geometric intuition. G. Cantor. Exiom of constructability. K. Gödel.

\footnotetext{
* Doutorado em Filosofia pela Pontifícia Universidade Católica do Rio de Janeiro Departamento de Filosofia/UFMT. <waltergomide@yahoo.com>.
} 
Não há dúvida de que a teoria dos conjuntos desenvolvida por Cantor é uma das construções intelectuais mais fascinantes da história do pensamento ocidental. Entretanto, não há também qualquer hesitação em dizer que os problemas fundacionais que subjazem na base da teoria são muito complexos, e há pouco consenso sobre a melhor forma de resolvê$\operatorname{los}^{1}$. Em síntese, responder à pergunta sobre quais são os fundamentos da teoria cantoriana não é uma tarefa fácil. Isto porque o próprio conceito de fundamento, em geral, parece não abarcar a construção da teoria dos conjuntos feita por Cantor, uma vez que esta construção se fez de forma intuitiva e partindo de concepções que nada se assemelham a noções bem "organizadas" da lógica ou do pensamento matemático em geral.

Primeiramente, para bem entender a origem da teoria dos conjuntos de Cantor, tenhamos em mente que ela surgiu embrionariamente nos estudos que Cantor realizou, por volta de 1870, sobre a unicidade da representação de funções contínuas por meio de série de funções trigonométricas (a tese de Fourier) ${ }^{2}$. Cantor concluiu que unicidade desta representação não era abalada se a função contínua em questão tivesse uma quantidade finita de pontos de descontinuidade ou, se a quantidade dos pontos for infinita, se este infinito de pontos de descontinuidade tivesse uma estrutura interna que "não incomodasse" a representação unívoca da função contínua. Na base dos estudos de Cantor sobre a unicidade da representação de funções contínuas sob a forma de funções trigonométricas, está o conceito de ponto-limite, que é definido por Cantor da forma seguinte:

[Um ponto limite] de $P$ [um conjunto de pontos da reta] é um ponto da linha reta situado de tal forma que, [qualquer que seja a sua vizinhança], nesta pode-se encontrar infinitos outros pontos de $P[\ldots]$ Por vizinhança de um ponto, entende-se qualquer intervalo que contenha tal ponto em seu interior (CANTOR in: KATZ [1993], p. 661).

Vê-se que o conceito de ponto-limite é um conceito topológicogeométrico e, sob tal condição, nos dá a dica de que a teoria cantoriana dos conjuntos, em seus aspectos embrionários, se assemelha a um estudo sistemático das propriedades dos números reais, vistos como pontos da reta.

A partir do conceito de ponto-limite, Cantor introduz a noção de conjunto derivado. Dado um conjunto $P$ de pontos da reta, podemos

1 Sobre a teoria de Cantor e seus fundamentos, ver DAUBEN, J. W: Georg Cantor. His Mathematics and Philosophy of the Infinite. Princeton University Press, New Jersey, [1979].

2 Sobre os trabalhos de Cantor em séries trigonométricas, ver "Über eine Ausdehnung eines Satzes aus der Theorie der trigonometrischen Reihen". Mathematische Annalen, [1872] 
formar o conjunto $P^{\prime}$ de seus pontos-limites. De $P^{\prime}$, podemos formar a sequência $P^{\prime \prime}, P^{\prime \prime \prime}, \ldots$ e, de forma geral, se $P^{n}$ for infinito, então haverá o conjunto $P^{n+1}$ de pontos limites (ver LAVINE [1998]). Mas não só isto: Cantor estendeu a sequência de conjuntos derivados até o infinito, uma vez que é introduzido o conjunto derivado

$$
P^{\propto}=\cap P^{k} \text {, para todo } k \text { finito. }
$$

Sendo $P^{\propto}$ um conjunto infinito, então existe o conjunto $P^{\propto+1}$ e, assim, a sequência de conjuntos

$$
P, P^{\prime}, P^{\prime \prime}, P^{\prime \prime \prime}, \ldots, P^{n}, P^{n+1}, \ldots, P^{\propto}, P^{\propto+1}, \ldots
$$

é concebível, partindo do pressuposto de que todos os seus termos sejam infinitos. Vê-se que tal sequência, com seus índices superiores, é o elemento ancestral da teoria de Cantor sobre os ordinais transfinitos e, portanto, pode-se afirmar que o infinito, nas mãos de Cantor, é abordado sobre o viés geométrico.

De fato, Cantor introduziu sua sequência dos números ordinais

$$
1,2,3, \ldots, n, \ldots, \omega, \omega+1, \omega+2, \ldots, \omega .2+1, \omega .2+2, \ldots, \omega . n, \ldots, \omega^{2}, \ldots
$$

a partir da tese que o primeiro ordinal transfinito $\mathrm{w}$, além de ser identificado com a compleição dos ordinais finitos $n$, situa-se infinitamente distante de qualquer número finito e pode ser compreendido como similar a um ponto ideal da teoria das funções analíticas de variáveis complexas (ver CANTOR [1883], §1, [4]-[5]). Sendo assim, a inspiração geométrica na elaboração que Cantor fez de sua teoria dos números transfinitos é clara.

Ainda na década de 70 dos oitocentos, Cantor demonstrou que o intervalo fechado de números reais entre 0 e 1 não poderia ser "listado", isto é, não há uma sequência cuja totalidade dos termos esgote a totalidade de números reais entre 0 e $1^{3}$. Daí Cantor concluiu a distinção entre conjuntos que são enumeráveis (os que podem ser "listados") e os nãoenumeráveis (os que não admitem ser postos sob a forma de uma "lista).

Mas não só isto se coloca como os trabalhos seminais da teoria dos conjuntos: em 1878, Cantor demonstrou que é possível uma bijeção entre o intervalo real $[0,1]$, um subconjunto do espaço $\mathbb{R}$, com a totalidade dos pontos de $\mathbb{R}^{n}$, sendo $n$ finito ${ }^{4}$. Mais precisamente, Cantor provou que

3 Ver CANTOR, G.: "Über Eine Eigenschaft des Inbegriffes aller reellen algebraischen Zahlen". In: Journal für reine und angewandte, Mathematik, 77, [1874].

4 Ver CANTOR: "Ein Beiträg für Mannigfaltigkeitslehre". In: Journal für reine und angewandte Mathematik, 84, [1878]. 
é possível estabelecer uma correspondência bijetiva entre espaços de dimensões $r$ e $p$, sendo $r \neq p$ e, com isto, demonstrou que há a mesma quantidade de pontos tanto em $\mathbb{R}^{r}$ quanto em $\mathbb{R}^{p}$, com $r \neq p$. Tal resultado que afirma que é possível emparelhar os pontos de espaços de dimensões diferentes causou espanto a Cantor que, admirado, expressou "vejo, mas não acredito" (CANTOR in: DUGAC [1976], p. 121). Entretanto, o espanto de Cantor não precisava ser tão grande: Dedekind, em um típico movimento de "intelecção inversa", esclareceu que tal bijeção só ocorre de maneira descontínua, posto que é impossível correlacionar espaços de dimensões distintas por meio de bijeções contínuas, uma vez que o número de dimensões de um espaço é um invariante topológico que se preservaria no caso de bijeções contínuas, o que não acontece com o "emparelhamento" demonstrado por Cantor. Sendo assim, a quantidade de pontos de um espaço é um "invariante" que se observa em relação a transformações não-contínuas entre espaços de dimensões diferentes.

Das observações críticas de Dedekind, Cantor percebeu que o invariante que deveria ser associado à bijeção entre espaços de dimensões distintas não seria de caráter topológico, mas sim de outra natureza: de fato, o que se mantém invariante na correspondência entre espaços lineares distintos é a sua potência ou o seu número cardinal (ver CANTOR [1895], § 1). Tem início assim, a partir do esclarecimento conceitual de um pretenso e extravagante resultado em topologia, a teoria dos conjuntos de Cantor.

Portanto, pode-se afirmar com tranquilidade que o contexto seminal da teoria dos conjuntos de Cantor foram seus trabalhos sobre representação de funções por meio de séries trigonométricas, correspondências entre espaços lineares e outros temas correlatos ${ }^{6}$. Em síntese, a teoria dos

5 O termo "intelecção inversa" (inverse insight) foi cunhado pelo Padre Bernard Lonergan e significa um insight ou intelecção que dirime problemas teóricos ao esclarecer ou ampliar um termo conceitual mediante uma nova abordagem. De fato, o espanto de Cantor ao se deparar com a existência da correspondência bijetiva entre espaços de dimensões diferente foi logo eliminado quando Dedekind esclareceu que tal correspondência era necessariamente nãocontínua e, portanto, em nada alteraria a tese já estabelecida que tal bijeção é impossível se considerarmos a bijeção como contínua. Sobre a noção de "intelecção inversa", ver LONERGAN, p.84, [2010].

6 De 1880 a 1884, em uma série de seis artigos intitulados "Über unendliche, lineare Punktmannigssfaltigskeiten" publicados pelos Mathematische Annalen, Cantor desenvolveu sua teoria sobre pontos-limites, assim seus conceitos de conjuntos derivados. Nestes artigos, Cantor estipula as diferenças topológicas entre os conjuntos enumeráveis e nãoenumeráveis, e tais distinções e todo o arcabouço teórico-conceitual que as fundamenta foram de precípua importância para o desenvolvimento da teoria cantoriana dos conjuntos, cujo ponto inicial se dá com a obra "Grundlagen einer allgemeinen Mannigfaltigkeitslehre", de 1883. 
conjuntos foi forjada em seus aspectos fundamentais a partir de um quadro conceitual topológico, e não a partir de intuições lógicas. Ao contrário de Frege, para quem um conjunto estaria atrelado à extensão de um conceito ou de uma propriedade ${ }^{7}$, para Cantor um conjunto é um agregado de objetos que são reunidos em nosso pensamento (CANTOR, [1895], §1), e esta reunião ou ajuntamento não precisa ser feito a partir de uma propriedade ou conceito lógicos que sirvam de "filtros" que retenham os objetos que pertencem ao agregado e dispensem os demais, aqueles que não possuem a propriedade definidora do agregado. Desta forma, pode-se dizer que a teoria dos conjuntos, em seus fundamentos, foi elaborada a partir de uma abordagem analógicogeométrica, e não lógica: os conjuntos são vistos como semelhantes a porções do espaço linear, isto é, são como intervalos abertos ou fechados de um espaço de $n$-dimensões, mas não como extensões de conceito.

Desta forma, uma vez que a noção de conjunto em Cantor não é de natureza lógica, não é de se esperar que a teoria dos conjuntos de Cantor se enquadre perfeitamente em um critério de logicidade, de tal forma que não haja, no interior da teoria cantoriana dos conjuntos, situações paradoxais que revelariam que os conceitos cantorianos não podem ser "encaixados" em uma perspectiva lógico-linguística, posto que são intrinsecamente de natureza geométrica ou topológica e, portanto, devem ser abordados analogicamente, e não de maneira lógica.

$\mathrm{E}$, de fato, tais situações existem: na teoria cantoriana dos conjuntos, existe o conceito de conjunto potenciação ("Potenz" - ver CANTOR, op. cit., §4) de um dado conjunto $A$, que nada mais é do que a totalidade dos subconjuntos de. De acordo com a noção de conjunto potenciação, para qualquer conjunto $X$, o número cardinal do conjunto potenciação de $X$ é maior que o número cardinal de $X$. No entanto, em um primeiro momento, a teoria dos conjuntos de Cantor permite que falemos de um maior conjunto de todos, como, por exemplo, a totalidade dos números ordinais. Chamemos tal conjunto de $\Omega$. Deduz-se que o número cardinal de $\Omega$ é o maior existente e que, por conseguinte, é maior que o cardinal do conjunto potenciação de $\Omega$. Mas isto é uma contradição, posto que, como já dito, é um teorema da teoria que qualquer conjunto $X$ tem número cardinal menor que o cardinal de seu conjunto potenciação ${ }^{8}$. De tal inconsistência, Cantor derivou a tese de que não há um maior

7 Ver Frege Grundlagen der Arithmetik. Eine logische matematische Untersuchung über den Begriff der Zahl, § 44-46, [1884].

8 Sobre a teoria de Cantor e seus paradoxos, ver HALLET, M. [1984]. Cantorian Set Theory and the Limitation of Size, Oxford: Clarendon Press. 
número cardinal, e o conceito de um conjunto de todos os conjuntos forma uma "multiplicidade inconsistente" que não pode ser fechada ou completa9.

Aparentemente, a contradição acima é uma prova inconteste de que o conceito de conjunto em Cantor é malformado logicamente. E, sem dúvida, isto é o caso simplesmente porque o conceito de conjunto não é lógico, mas analógico: o conjunto $\Omega$ (ou outros similares) que gera a contradição não é de fato, como já mencionado no parágrafo acima, um conjunto, mas uma multiplicidade inconsistente, um agregado que não pode ser tomado como completo ou encerrado; é algo análogo a um intervalo aberto da reta real ${ }^{10}$. Assim, a sua incapacidade de se adequar aos critérios lógicos de consistência é perfeitamente compreensível, posto que se trata de um conceito que encontra sua fundamentação na síntese geométrica, e não na análise lógica.

Os paradoxos gerados no interior da teoria cantoriana ocasionaram a busca por uma fundamentação lógica da noção de conjunto, e esta busca encontrou guarida na tentativa de apresentar a teoria de Cantor sob a forma axiomática.

Em 1908, Zermelo apresenta sua versão axiomatizada para a teoria de Cantor ${ }^{11}$. Com sua apresentação "domesticada" da noção de conjunto ${ }^{12}$ e da noção de transfinito, esperava-se que os velhos paradoxos da teoria de Cantor, frutos de uma concepção naif e, por conseguinte, geométrica de conjunto, fossem eliminados. De fato, a versão axiomatizada de Zermelo, a qual pode ser enriquecida pelo axioma da escolha, acréscimo este que gera o que conhecemos com a axiomática

9 Segundo Cantor:

"Uma multiplicidade pode ser tal que a tese de que a "união" de todos os seu elementos leva a uma contradição, e neste caso é impossível ver tal multiplicidade como uma unidade, como "algo completo". Tais multiplicidades eu denomino de infinitos absolutos ou multiplicidades inconsistentes" (ver CANTOR in: "Cantor, God, and Inconsistent Multiplicities", THOMASBOLDUC, Aaron R, [2016] - tradução livre feita por Walter Gomide a partir da versão em inglês presente em Thomas-Bolduc).

10 Em geral, um intervalo é uma porção conexa de pontos da reta real. Se os pontos extremos desta porção estão excluídos do intervalo, então diz-se que temos um intervalo aberto (ver GEMIGNANI, M. C. Elementary Topology. New York: Dover, [1990]).

11 Ver Zermelo, E. [1908]: "Untersuchungen über die Grundlagen der Mengenlehre, I", Mathematische Annalen, 65, p. 261-281.

12 O adjetivo "domesticado" usada em relação à apresentação que Zermelo faz da teoria dos conjuntos se deve, em especial, a presença do axioma da separação, que afirma que, dado um domínio $X$ de objetos, então há o conjunto $Y$ formado por todos os objetos de $X$ que satisfazem uma propriedade definida em $X$. Assim, a noção de uma propriedade bem definida que filtra os elementos de um dado conjunto também bem definido para a formação de um novo conjunto introduz na teoria dos conjuntos um claro limite naquilo que podemos conceber como conjunto: as ilimitadas e indômitas multiplicidades inconsistentes de Cantor saem de cena na formulação de Zermelo (ver Russell, § 104, [1903]). 
de Zermelo-Fraenkel $\mathrm{ZFC}^{13}$, inibe o surgimento de conjuntos absurdamente grandes, os quais seriam as "causas" do surgimento de tais paradoxos. Desta forma, paira sobre a construção axiomática de ZFC a tese de que "conjunto" é um recorte de objetos que tem um complementar em relação a um universo de objetos, e esta concepção está dada a partir da tese de que a definição de um conjunto se dá por uma propriedade que deve ser consistentemente expressa na linguagem lógica ${ }^{14}$.

Depreende-se, então, que a idéia diretriz por trás da axiomática da teoria dos conjuntos, que pode ser vista como uma tentativa de apaziguar as intuições originais de Cantor, é a de que a lógica ou o pensamento analítico são a fonte de onde sai o conceito de conjunto, sendo tal conceito tomado inclusive na sua radicalidade de conjunto infinito. A teoria cantoriana dos conjuntos, apesar de seu contexto de origem ser o universo topológico-geométrico, de fato esconde a sua base lógico-analítica, e caberia a teoria ZFC deixar esta base em evidência.

13 A teoria ZFC consiste na teoria inicial elaborada em 1908 por Zermelo, incrementado pelos axiomas da regularidade, da substituição e da escolha. Mais precisamente, os axiomas de ZFC são os seguintes:

a) Axioma da Existência:

Há um conjunto que não tem elementos (o conjunto vazio);

b) Axioma da Extensionalidade:

Se todo element de $X$ é elemento de $Y$, e se todo elemento de $Y$ é elemento de $X$, então $X=Y$;

c) Axioma (Esquema) da Separação:

Seja $P(x)$ uma propriedade de $\mathrm{x}$. Para qualquer conjunto $A$, existe um conjunto $B$ tal que $x \in B$ se, e somente se, $x \in A$ e $P(x)$ é o caso;

d) Axioma do Par:

Para quaisquer $A$ e $\mathbf{B}$, existe um conjunto $C$ tal que $x \in C$ se, e somente se, $x=A$ ou $x=B$;

e) Axioma da União:

Para qualquer conjunto $S$, existe um conjunto $U$ tal que $x \in U$ se, e somente se, $x \in A$ para algum $A \in S$.

f) Axioma do Conjunto Potência:

Para qualquer conjunto $\mathrm{S}$, existe um $P$ tal que $X \in P$ se, e somente se, $X \subseteq S$;

g) Axioma do Infinito:

Existe um conjunto $X$ tal que $\varnothing \in X$, e se $Y \in X$, então $\{Y\} \in X$;

h) Axioma (Esquema) da Substituição:

Seja $P(x, y)$ uma propriedade tal que, para todo $x$, há um único y para o qual $P(x, y)$ é o caso. Para todo $A$ conjunto $A$ existe um conjunto $B$ tal que, para todo $x \in A$, existe um $y \in B$ para o qual $P(x, y)$ é o caso;

i) Axioma da Escolha:

Para toda família $X$ de conjuntos distintos dois a dois, existe um conjunto $Y$ que contém exatamente um elemento em comum com cada um dos conjuntos da família $X$.(ver FRAENKEL, A. [1961])

14 Em 1923, Thoralf Skolem propôs que a propriedade bem definida que aparece no axioma da separação deveria ser uma expressão bem formada da lógica de primeira ordem (ver SKOLEM, T., [1923], in: [1977], "Some remarks on axiomatized set theory", From Frege to Gödel: A Source Book in Mathematical Logic, 1879-1931 (3rd ed.). Cambridge, Massachusetts: Harvard University Press, p. 290-301. 
Mas, para tanto, a apresentação intuitiva e "quase-geométrica" da teoria de Cantor teria de ser reformulada. Nesta reformulação, a introdução dos números transfinitos, em especial os ordinais, como análogos aos pontos ideias da geometria projetiva, deve ser substituída por uma outra em que somente os postulados de ZFC sejam usados. Assim, a partir da década de 1930, surge a concepção cumulativa dos conjuntos. Definida a partir de recursão transfinita, o universo cumulativo de conjuntos, conhecido como universo de Von Neumann, se define como se segue ${ }^{15}$ :

a) O estágio $V_{0}$ é o conjunto vazio:

$$
V_{0}=\varnothing ;
$$

b) Para qualquer ordinal $\beta$, o estágio $V_{\beta+1}$ é definido como:

$$
V_{\beta+1}=\wp\left(V_{\beta}\right) ;
$$

C) Para qualquer ordinal limite $\lambda$, temos que:

$$
V_{1}=U_{\beta<\lambda} V_{\beta} .
$$

Em geral, $V=\bigcup_{\alpha} V_{\alpha}$, em que $\alpha \in$ Ord, a classe de todos os ordinais.

A hierarquia $V$ é gerada a partir dos axiomas de ZFC (mas não somente) e a partir do conjunto vazio. Esta hierarquia está construída sobre a tese de que qualquer estágio de conjuntos concebível, por maior que seja, não é introduzido de forma postulada, como nos trabalhos originais de Cantor, mas como resultados de operações bem definidas realizadas em níveis inferiores da hierarquia. Com isto, cada ordinal transfinito se legitima a partir dos níveis inferiores da hierarquia e, com isto, a consistência da introdução destes ordinais é função imediata da própria consistência de ZFC.

Desta forma, os estágios da hierarquia nos dão os conjuntos disponíveis e "bem gerados" pelas operações e axiomas definidos em ZFC, sem que entremos no mérito se há ou não propriedades ou conceitos que se associem a tais conjuntos. Dito de outra maneira, não se pergunta se os conjuntos gerados estão ou não associados a alguma propriedade que se possa ser expressa na linguagem lógica. De fato, os níveis sucessivos da hierarquia $V$ são compostos de todos os subconjuntos que existem no nível anterior, mas não se tem a preocupação de associar a tais subconjuntos propriedades expressas na linguagem lógica; de alguma

15 Apesar de ser conhecido como universo de Von Neumann, a classe $V$ dos conjuntos cumulativos foi proposta inicialmente por Ernst Zermelo, em 1930 (ver ZERMELO, [1930]: "Über Grenzzahlen und Mengenbereiche: Neue Untersuchungen über die Grundlagen der Mengenlehre". Fundamenta Mathematicae, 16: 29-47. 
maneira, portanto, a hierarquia $V$ preza mais uma pressuposta intuição pura do que seja conjunto do que uma preocupação fundacional em que a noção de conjunto se depreenda de intuições lógico-analíticas

Esta preocupação lógico-analítica e construtiva, por assim dizer, é incorporada à hierarquia $V$ quando se introduz o postulado de que todo conjunto tem relacionado a si uma propriedade expressa na linguagem de primeira ordem (ver nota 13); e, de fato, tal postulado foi introduzido por Gödel, em 1938, a fim de provar a validade tanto do axioma da escolha quanto da hipótese do contínuo em domínios compostos de conjuntos construtíveis ${ }^{16}$. Gödel, no referido trabalho, apresentou a tese de que todo conjunto é definível por expressões de primeira ordem com parâmetros e quantificadores que atuam nos estágios anteriores da hierarquia. Tal tese, conhecida como axioma da construtibilidade, é expressa como $V=L$, em que $L$ é a hierarquia dos universos construtíveis cujo estágiozero é composto somente do conjunto vazio. A saber, a hierarquia $L$ dos conjuntos construtíveis e a seguinte:

a) O estágio $L_{0}$ é o conjunto vazio:

$$
L_{0}=\varnothing \text {; }
$$

b) Para qualquer ordinal $\beta$, o estágio $L_{\beta+1}$ é definido como:

$$
L_{\beta+1}=\operatorname{Def}\left(L_{\beta}\right),
$$

em que: $\operatorname{Def}(\mathrm{X})=\left\{\left\{\mathrm{y} / \mathrm{y} \in \mathrm{X}\right.\right.$ e $\left.<X, \in>\vDash \Phi\left(y, z_{1}, \ldots, z_{n}\right)\right\} / \Phi$ é uma fórmula de primeira ordem e $\left.z_{1}, \ldots, z_{n} \in X\right\}^{17}$;

c) Para qualquer ordinal limite $\lambda$, temos que:

$$
L_{\lambda}=\bigcup_{\beta<\lambda} L_{\beta} .
$$

Em geral, $V=U_{\alpha} L_{\alpha}$, em que $\alpha \in$ Ord, a classe de todos os ordinais.

É digno de nota 0 fato de que o axioma $V=L$ insere na teoria dos conjuntos a mais clara intuição lógico-analítica e construtivista: os objetos matemáticos, em especial os conjuntos, são objetos construídos a partir de propriedades bem definidas. Com o axioma da construtibilidade, qualquer resíduo topológico-geométrico que pudesse "contaminar" a pureza lógica e construtiva dos conjuntos é eliminado.

Ao consideramos somente o que é proposto pela hierarquia $V$ simpliciter, temos que os conjuntos são entidades "matemáticas" que

16 Ver Gödel, [1938]: “The Consistency of the Axiom of Choice and of the Generalized Continuum Hypothesis". Proceedings of the National Academy of Sciences, U.S.A. [s.l.: s.n.] 24, p. 556-557.

17 Em linhas gerais, Def $(X)$ nos dá todos os conjuntos y que pertencem ao conjunto $X$ e que satisfazem, no conjunto $X$ visto como um modelo, a fórmula $\Phi\left(y, z_{1}, \ldots, z_{n}\right)$; os parâmetros $z_{1}, \ldots, z_{n}$ também pertencem a $X$. 
estão bem fundadas nos axiomas de ZFC e na existência, postulada por tais axiomas, do conjunto vazio. O fato de haver tais estágios e operações nos dá um limite para o que deve ser tomado como o tamanho dos conjuntos, mas não impede que vejamos os conjuntos como baseados em uma síntese geométrica que nada tem a ver com a lógica. No entanto, com o postulado de $V=L$, os conjuntos, em qualquer estágio da hierarquia, passam a ser associados com expressões bem definidas da lógica de primeira ordem e, com isto, a natureza lógico-analítica da noção de conjunto é colocada de forma imediata.

No entanto, este desiderato lógico-analítico não parece ser a rota ou o caminho de Cantor na formulação de sua teoria dos conjuntos e sobre os números transfinitos. Em Cantor, como já foi mostrado aqui, os conjuntos surgem de contextos geométricos ou topológicos, e a introdução de seus cardinais ou ordinais transfinitos se faz de forma postulacional, sem a preocupação prévia de que os conjuntos ou os números transfinitos sejam dados como lógicos. Assim, é de se esperar que esta incongruência entre a atitude original de Cantor e aquela que orientou Gödel ao postular que $V=L$ aparecesse em algum momento no desenvolvimento ulterior da teoria dos conjuntos. Veremos em seguida que a noção de cardinal mensurável traz em si esta incompatibilidade entre a visão sintéticogeométrica de Cantor e a concepção lógico-analítica de conjunto expressa em $V=L$.

Como já foi dito, a teoria de Cantor dos conjuntos surgiu em contextos topológico-geométricos, e este fato implica que os conceitos de infinito e conjunto têm, em algum grau, uma natureza extensiva: os conjuntos de Cantor guardam uma forte analogia com os abertos topológicos. De fato, os conjuntos cantorianos são vistos combinatoriamente como um agregado de elementos quaisquer, e tal concepção guarda uma estrita similaridade com a idéia de uma região do espaço composta por uma reunião de pontos. Em Cantor, os conjuntos não são de antemão compreendidos como extensão de conceitos, mas como totalidades de objetos quaisquer que podem ser agregados pelo pensamento. Daí a inexistência em Cantor do conjunto vazio, o qual aparece na teoria de ZFC a partir do axioma da existência (ver nota 13). Assim, uma das perdas que a tese $V=L$ pode implicar, uma vez que tal tese insere os conjuntos como entidades matemáticas construídas a partir de critérios lógico-linguísticos, é a desconsideração das propriedades geométricas ou extensivas que os conjuntos possuam em função de sua analogia com as regiões do espaço - o caráter topológico dos conjuntos.

Uma das propriedades extensivas que se esperam que os conjuntos possam ter é a mensurabilidade em sentido geral. Isto porque é natural que se espere que os conjuntos, uma vez que sejam agregados com 
tamanho e extensão possivelmente infinitos, admitam ser avaliados com alguma medida. De maneira geral, dado um conjunto $S$ qualquer, podemos perguntar se existe uma função-medida $\mu: \wp(S) \rightarrow[0,1] \mathrm{com}$ as seguintes características (ver KANAMORI, p. 23, [2003]):

i) $\mu(S)=1$

ii) $\mu(\{x\})=0$, para todo $x \in S$;

iii) para conjuntos distintos $\left\{X_{\alpha} / \alpha<\lambda\right\} \subseteq \wp(S)$, $\mu\left(\bigcup_{\lambda} X_{\lambda}\right)=\mu\left(\Sigma_{\lambda} \mu\left(X_{\lambda}\right)\right)$, sendo $\lambda$ um ordinal limite.

Cabe então a pergunta se há cardinais infinitos mensuráveis, isto é, se é possível definir nos conjuntos infinitos uma medida como apresentada acima ${ }^{18}$. Sendo a medida ou a mensuração uma propriedade inerente ao que é extenso e que tenha tamanho, como uma região do espaço ou o seu análogo conjuntístico, é desejado que o tratamento lógico-construtivo que os conjuntos recebem com a tese $V=L$ não interfira na atribuição de uma medida aos conjuntos. Mas não é bem isto o que ocorre: o matemático americano Dana Scott, em 1961, demonstrou que se existem em ZFC cardinais mensuráveis, então $V \neq L .{ }^{19}$ Tal resultado impõe um forte limite à concepção de que os conjuntos são imagens extensionais de expressões de primeira ordem definidas em ZFC. Assim, o teorema de Dana Scott nos diz que o caráter extensivo dos conjuntos, que nos dá a intuição de que os mesmos podem ser medidos, é perdido se pretendermos dar aos conjuntos uma abordagem lógico-construtiva.

Com o teorema de Dana Scott, surge a questão de saber qual o fundamento intuitivo da noção de conjunto e, em especial, do conceito de conjunto infinito. Como já foi observado, a teoria de Cantor, em seu contexto original, não se preocupava com os fundamentos lógicos dos novos conceitos elaborados em seu escopo. A noção de conjunto e os seus correlatos como números ordinal e cardinal eram vistos como intuitivos, como objetos cuja fundamentação bastavam a intuição ou o pensamento. Também é digno de nota que Cantor derivou os conceitos de cardinal e ordinal de situações em que a intuição geométrica era muito mais prevalente do que a intuição lógica: Cantor não se incomodava com propriedades ou conceitos de cuja extensão os conjuntos seriam a imagem, mas estava preocupado em medir ou contar o infinito; e, neste sentido, provavelmente concordaria ou não teria problemas em atribuir

18 Cabe notar que os cardinais finitos ou enumeráveis têm trivialmente medida zero, e a função medida que os avalia não satisfaz a condição i) da definição da função $\mu$. De fato, a questão de saber se há cardinais mensuráveis torna-se um tema não trivial quando se admite que os cardinais $\kappa$ a serem medidos são aqueles que possuem certos subconjuntos $X$ (ultrafiltros) que possuem a propriedade de $\mu(X)=1$.

19 Ver Scott, D. [1961]: "Measurable Cardinals and Constructible Sets". BAPS, 9, p. 521-524, XVII, 44, 49. 
uma medida para os seus conjuntos - de fato, os cardinais transfinitos de Cantor são as medidas ou os tamanhos do infinito.

Por conseguinte, a tese de Gödel de que $V=L$ é uma adaptação da teoria de Cantor para uma visão filosófica pautada na precedência da linguagem lógica em relação aos objetos matemáticos. Estes objetos matemáticos, que no realismo de Cantor existem por si mesmos e são tratados intuitivamente, devem ser, sob a perspectiva do construtivismo lógico implícito em $V=L$, vistos como bem fundamentados em uma linguagem lógica livre de contradições. Não que Cantor se sentisse à vontade com paradoxos ou contradições, mas simplesmente postulava que os objetos contraditórios não devem ser completados e apresentados, em qualquer linguagem, como prontos ou bem definidos: nenhuma linguagem, seja esta lógica ou analógico-geométrica (sintética, para usar um termo kantiano) está apta para lidar com o absolutamente infinito (as multiplicidades inconsistentes, segundo Cantor), e tal fato não se deve a peculiaridades da linguagem em questão, mas sim a natureza última dos objetos contraditórios, que são como limites para qualquer tentativa humana de delimitar o que é essencialmente não-delimitável.

\section{Referências}

1. CANTOR, G. [1895/97]. "Beiträge zur Begründung der transfiniten Mengenlehre". In: Cantor [1932], 282-351. English trans. in Cantor. Contributions to the founding of the theory of transfinite numbers. New York: Dover, [1955].

2. CANTOR, G. [1878]. "Ein Beiträg für Mannigfaltigkeitslehre". In: Journal für reine und angewandte Mathematik,84, [1878].

3. CANTOR, G. [1932]. Gesammelte Abhandlungen mathematischen und philosophischen Inhalts, E. Zermelo (ed.), Berlin: Springer. Reprint Hildesheim: Olms, [1966].

4. CANTOR, G. [1883]. Grundlagen einer allgemeinen Mannigfaltigkeitslehre, Leipzig: B. G. Teubner. In Cantor 1932, 165-208. English in Ewald [1996]. v. 2.

5. CANTOR, G. [1872]. "Über Eine Ausdehnung eines Satzes aus der Theorie der trigonometrischen Reihen". Mathematische Annalen".

6. CANTOR, G. [1874]. "Über Eine Eigenschaft des Inbegriffes aller reellen algebraischen Zahlen". In: Journal für reine und angewandte Mathematik, 77.

7. CANTOR, G. [1892]. "Über eine elementare Frage der Mannigfaltigkeitslehre," Jahresbericht der Deutschen MathematikerVereinigung, 1: 75-78. In Cantor (1932), 278-280. English trans. In: Ewald [1996], v. 2.

8. CANTOR, G. [1884]. "Über unendliche, lineare Punktmannichfaltigkeiten, 6," Mathematische Annalen, 23: 453-488. In: Cantor [1932], 210-244.

9. DAUBEN, J. W. Georg Cantor. His Mathematics and Philosophy of the Infinite. Princeton University Press, New Jersey, [1979]. 
10. DUGAC, J. [1976]. Richard Dedekind et les Fondements des Mathematiques. J. Vrin, Paris.

11. EWALD, W. B. [1996]. From Kant to Hilbert: A Source Book in the Foundations of Mathematics. Oxford: Oxford University Press. 2 v.

12. FRAENKEL, A. [1961]. Abstract Set Theory. North Holland Publishing Co, Amsterdam.

13. FREGE, G. Die Grundlagen der Arithmetik: eine logisch-mathematische Untersuchung über den Begriff der Zahl, Breslau: W. Koebner, [1884]. Translated as The Foundations of Arithmetic: A logico-mathematical enquiry into the concept of number, by J. L. Austin. Oxford: Blackwell, 2nd ed., rev. [1974].

14. GEMIGNANI, M. C. Elementary Topology. New York: Dover, [1990].

15. GÖDEL, K. [1938]. "The Consistency of the Axiom of Choice and of the Generalized Continuum Hypothesis". Proceedings of the National Academy of Sciences, U.S.A. [S.l.: s.n.] 24, p. 556-557.

16. HALLET, M. [1984]. Cantorian Set Theory and the Limitation of Size. Oxford: Clarendon Press.

17. KANAMORI, A. [2003]. The Higher Infinite. Second Edition. Springer Monographs in Mathematics, New York: Springer.

18. KATZ, V. A History of Mathematics. An Introduction. New York: Harper Collins College Publishing Co.

19. LAVINE, S. [1994]. Understanding the Infinite, Cambridge, MA: Harvard University Press.

20. LONERGAN, B. Insight - A Study of Human Understanding [1957]. Tradução para o português [2010]: insight - Um estudo sobre o Entendimento Humano. Tradução de Mendo Castro Henriques e Artur Mourão. São Paulo: É Realizações Editora, [2010].

21. RUSSEL, B. [1903]. The Principles of Mathematics, Cambridge, University Press. Reprint of the 2nd edn. [1937]: London: Allen \& Unwin, [1948].

22. SCOTT, D. [1961]. "Measurable Cardinals and Constructible Sets". BAPS 9. 521-524, XVII, 44, 49.

23. SKOLEM, T. [1923], in: [1977], "Some remarks on axiomatized set theory", From Frege to Gödel: A Source Book in Mathematical Logic, 1879-1931 (3rd ed.), Cambridge, Massachusetts: Harvard University Press, p. 290-301.

24. THOMAS-BOLDUC, A.R. [2016]. "Cantor, God, and Inconsistent Multiplicities". In: Studies in Logic, Grammar and Rhetoric, 44 (1): 133-146.

25. ZERMELO, [1930]. "Über Grenzzahlen und Mengenbereiche: Neue Untersuchungen über die Grundlagen der Mengenlehre". Fundamenta Mathematicae, 16: 29-47;

26. ZERMELO, E. [1908]. "Untersuchungen über die Grundlagen der Mengenlehre, I", Mathematische Annalen, 65: 261-281.

\section{Endereço postal:}

Universidade Federal de Mato Grosso,

ICHS- Departamento de Filosfia

Boa Esperança - Cuiabá, MT, Brasil

Data de recebimento: 02-11-2016

Data de aceite: 07-11-2016 